\title{
A RIGIDITY THEOREM FOR SPECIAL FAMILIES OF RATIONAL FUNCTIONS
}

\author{
Greg Markowsky \\ Monash University, Department of Mathematical Sciences \\ Victoria 3800, Australia; gmarkowsky@gmail.com
}

\begin{abstract}
We study the question of whether for a given nonconstant holomorphic function $f$ there is a pair of domains $U, V$ such that $f$ is the only nonconstant holomorphic function with $f(U) \subseteq V$. We show existence of such a pair for several classes of rational functions, namely maps of degree 1 and 2 as well as arbitrary degree Blaschke products. We give explicit constructions of $U$ and $V$, where possible. Consequences for the generalized Kobayashi and Carathéodory metrics are also presented.
\end{abstract}

\section{Introduction}

Let $U=\left\{\infty, e^{\pi i / 6}, e^{\pi i / 3}, e^{\pi i / 2}, e^{\pi i / 6}\right\}^{c}$, with this and all future complements taken in the Riemann sphere, $\hat{\mathbf{C}}=\mathbf{C} \cup\{\infty\}$. Let $f(z)=z^{2}$ and $V=f^{-1}(U)$. In [3] and $[4$, pp. 162, 171] it was shown that if $g$ is a nonconstant holomorphic function on $V$ such that $g(V) \subseteq U$ then $g=f$. This example is motivated by the subjects of [3] and [4, Sec.'s 8.2, 9.2], the generalized Kobayashi and Carathéodory metrics on plane domains, which are defined in terms of the family of holomorphic maps from one domain to another(definitions given in Section 5 below). When a pair of domains has only one nonconstant holomorphic function between them, the metrics are explicitly computable.

The object of this paper is to study this question in more generality. That is, loosely speaking, we study the question of whether for a given nonconstant holomorphic function $f$ there is a pair of domains $V, U$ such that (a) $f(V) \subseteq U$ and (b) if $g$ is a nonconstant holomorphic function such that $g(V) \subseteq U$ then $g=f$. In this paper we will restrict to rational functions; the question for arbitrary functions will be discussed briefly in the final section.

We will begin by making the problem a bit more concrete. To abbreviate the language somewhat, in what follows we will refer to a nonconstant holomorphic function as a map. If the domain $V$ admits bounded maps, then clearly there will be many different maps into any image domain $U$. If $V^{c}$ contains a continuum, then there will be bounded maps on $V$, so it is necessary for $V^{c}$ to be small. We will see that in our results it is convenient and sufficient to consider only finite sets for $U^{c}, V^{c}$. If $U^{c}$ has 1 or 2 points then proper transformations of $e^{h}$ for any holomorphic function $h$ on $V$ will map into $U$. We therefore restrict our attention to the case where $U^{c}$ contains at least 3 points. In this case any map $f$ from $V$ to $U$ contains no essential singularities on the sphere, and thus is the restriction of a rational function to $V$.

doi:10.5186/aasfm.2012.3717

2010 Mathematics Subject Classification: Primary 30E99.

Key words: Complex variables, rational functions, generalized Kobayashi metric, generalized Caratheodory metric. 
We must have $f^{-1}\left(U^{c}\right) \subseteq V^{c}$, and we see that it is enough to consider only the case $V^{c}=f^{-1}\left(U^{c}\right), V=f^{-1}(U)$. In light of this, for any rational function $f$ and domain $U$ define

$$
\mathcal{H}_{f}(U)=\left\{h: h \text { is a map from } f^{-1}(U) \text { to } U\right\} .
$$

Our goal is now to find a domain $U$ such that $\mathcal{H}_{f}(U)=\{f\}$, for a given $f$. Suppose that we have accomplished this for some $f$, so that $\mathcal{H}_{f}(U)=\{f\}$. If $g$ is a different rational function such that $g=\phi_{1} \circ f \circ \phi_{2}$, where $\phi_{1}, \phi_{2}$ are Möbius transformations, then it is not hard to see that $\mathcal{H}_{g}\left(\phi_{1}(U)\right)=\{g\}$. In this case we will say that $f$ and $g$ are Möbius equivalent, and it is clear that we need only study our problem on Möbius equivalence classes. In the next section we give complete solutions when $f$ is of degree 1 or 2 . In Section 3 we prove an existence theorem for Blaschke products of arbitrary degree. This theorem guarantees the existence of a suitable domain $U$, but is nonconstructive. In Section 4 we give explicit solutions for the functions $f(z)=z^{n}$. Section 5 gives some applications of these results to the generalized Kobayashi and Carathéodory metrics. Finally, Section 6 gives a few further problems which may be of interest.

\section{Rational functions of degree 1 and 2}

A rational function of degree 1 is a Möbius transformation and is therefore clearly Möbius equivalent to the identity. The following proposition therefore gives the complete solution in the degree 1 case.

Proposition 1. Let $f(z)=z$ and $E=\{-1,0,1, \infty, 2 i\}$. Then $\mathcal{H}_{f}\left(E^{c}\right)=\{f\}$.

Proof. Suppose $h$ is a map in $\mathcal{H}_{f}\left(E^{c}\right)$. Since $E$ has more than 3 points, $h$ extends to a rational function on the sphere. Let the degree of $h$ be denoted $d$. By the Riemann-Hurwitz formula, $h$ has $2 d-2$ critical points counting multiplicity, so the size of $h^{-1}(E)$ is at least $5 d-(2 d-2)=3 d+2$. Since we must have $h^{-1}(E) \subseteq E$, we conclude $d=1$, and $h$ is a Möbius transformation. Set $h(z)=\frac{a z+b}{c z+d}$. The set $h(\{-1,0,1, \infty\}) \cup\{-1,0,1, \infty\}$ must contain at least 3 points, which means $h(\mathbf{R})=\mathbf{R}$ and thus we can take $a, b, c, d$ to all be real and we have $h(2 i)=2 i$. Equating real and imaginary parts in $a(2 i)+b=2 i(c(2 i)+d)$ shows that $a=d$, $b=-4 c$. If $a=d=0$, then $h(z)=\frac{-4}{z}$, which does not map $E$ to $E$. We can therefore set $a=d=1$, and $h=\frac{z+b}{-4 b z+1}$. This maps $\infty$ to $\frac{1}{-4 b}$ and 0 to $b$. The only choice for $b$ such that $b, \frac{1}{-4 b} \in E$ is $b=0$, so that $h$ is the identity.

Remark. In this proposition it was necessary that $E$ contain at least 5 points. This can be seen by noting that $f(z)=\frac{a}{z}$ is a nonconstant self-map of $\{0,1, \infty, a\}^{c}$ for any $a$.

It was shown in [3] that $\mathcal{H}_{z^{2}}\left(\left\{\infty, e^{\pi i / 6}, e^{\pi i / 3}, e^{\pi i / 2}, e^{\pi i}\right\}^{c}\right)=\left\{z^{2}\right\}$. The following proposition shows that this extends to all rational functions of degree 2.

Proposition 2. Suppose $f(z)$ is a rational function of degree 2. Then there is a set $E$ of 5 points such that $\mathcal{H}_{f}\left(E^{c}\right)=\{f\}$.

Proof. Since $f$ is of degree 2, topological considerations or direct calculations show that $f$ has exactly two critical values. That is, there are two points $u, v$ such that $f^{-1}(u)$ and $f^{-1}(v)$ are singletons. We see that $f$ is Möbius equivalent to a degree 2 rational function with $f^{-1}(0)=\{0\}$ and $f^{-1}(\infty)=\{\infty\}$. We may therefore 
take $f(z)=c z^{2}$, and again via Möbius equivalence we may assume $c=1$. The case $f(z)=z^{2}$, as has been mentioned, appeared in [3] and [4]. Alternatively, Theorem 1 below applies.

Remark. The remark following Proposition 1 shows that again $E$ must have at least 5 points, since if $E$ has 4 or less points $\mathcal{H}_{f}\left(E^{c}\right)$ will always contain Möbius transformations.

\section{Functions Möbius equivalent to Blaschke products}

The following theorem, together with the ensuing corollary, give a solution for all Möbius equivalence classes containing a Blaschke product of degree at least 3 .

Theorem 1. Suppose that $f(z)=\lambda \prod_{j=1}^{d} \frac{z-a_{j}}{1-\overline{a_{j}} z}$, where $\left|a_{1}\right|, \ldots,\left|a_{d}\right|<1,|\lambda|=1$, and $d \geq 3$. Then there is a set $E$ of $2 d+1$ points such that if $U^{c}=E$, then $\mathcal{H}_{f}(U)=\{f\}$.

Proof. $E$ will be $\infty$ together with $2 d-1$ points $p_{1}, \ldots, p_{2 d-1}$ chosen arbitrarily on the unit circle, as well as another point $p_{2 d}$, also on the unit circle, to be determined later. Set $U=E^{c}$, and suppose that $h$ is a map from $f^{-1}(U)$ to $U$. Since $U^{c}$ has more than 3 points, $h$ extends to a rational function on the sphere. Let the degree of $h$ be denoted $d_{h}$. By the Riemann-Hurwitz formula, $h$ has $2 d_{h}-2$ critical points counting multiplicity, and thus the size of $h^{-1}(E)$ is at least $d_{h}(2 d+1)-\left(2 d_{h}-2\right)=d_{h}(2 d-$ $1)+2$. On the other hand, we must have $h^{-1}(E) \subseteq f^{-1}(E)$, and $f^{-1}(E)$ can have at most $d(2 d+1)$ points. Thus, $d(2 d+1) \geq d_{h}(2 d-1)+2$, which implies that $d_{h} \leq d$. Since $f$ is a Blaschke product, $f^{-1}\left(\left\{p_{1}, \ldots, p_{2 d}\right\}\right) \subseteq\{|z|=1\}$. Any members of $h^{-1}\left(\left\{p_{1}, \ldots, p_{2 d}\right\}\right)$ not on the unit circle must therefore lie in $f^{-1}(\infty)$, which contains at most $d$ points. Since $2 d \geq d+3$, we can find points $a, b, c \in\left\{p_{1}, \ldots, p_{2 d}\right\}$ such that $h^{-1}(\{a, b, c\}) \subseteq\{|z|=1\}$. Since Möbius transformations act transitively on the set of circles and lines, $h$ is Möbius equivalent to a rational function $\tilde{h}$ of degree $d_{h}$ such that $\tilde{h}^{-1}(\{0,1, \infty\}) \subseteq \mathbf{R} . \tilde{h}$ must be of the form

$$
\tilde{h}(z)=k \frac{\left(z-\alpha_{1}\right) \ldots\left(z-\alpha_{d_{h}}\right)}{\left(z-\beta_{1}\right) \ldots\left(z-\beta_{d_{h}}\right)}
$$

and since $\tilde{h}^{-1}(\{0,1, \infty\}) \subseteq \mathbf{R}$ we see that $k, \alpha_{1}, \ldots, \alpha_{d_{h}}, \beta_{1}, \ldots, \beta_{d_{h}} \in \mathbf{R}$. Thus, $\tilde{h}(\mathbf{R} \cup\{\infty\}) \subseteq \mathbf{R} \cup\{\infty\}$, and it follows that $h(\{|z|=1\}) \subseteq\{|z|=1\}$. This implies that $h^{-1}(\infty) \cap\{|z|=1\}=\emptyset$, so that $h^{-1}(\infty) \subseteq f^{-1}(\infty)$. Let $k$ be a Blaschke product having the same poles of the same order as $h$. Then, since $|k(z)|=|h(z)|=1$ on the unit circle, by the reflection principle $k$ and $h$ have the same zeroes of the same orders as well, so that $\frac{h(z)}{k(z)}$ is a map from the sphere to itself which is never 0 or $\infty$ and is hence constant. We conclude that $h$ is itself a Blaschke product. Write $h(z)=\lambda^{\prime} \prod_{j=1}^{d_{h}} \frac{z-b_{j}}{1-\overline{b_{j}} z}$. $h$ may only have poles at poles of $f$, so there are only finitely many possibilities for the function $\prod_{j=1}^{d_{h}} \frac{z-b_{j}}{1-\overline{b_{j}} z}$. Recall that $p_{1}, \ldots, p_{2 d-1}$ were fixed arbitrarily, while $p_{2 d}$ is at this point still a variable point on the unit circle. Considering the cardinality of the sets, it is clear that $h^{-1}\left(\left\{p_{1}, \ldots, p_{2 d-1}\right\}\right) \nsubseteq f^{-1}\left(p_{2 d}\right)$, so there is at least one point $u$ in $f^{-1}\left(\left\{p_{1}, \ldots, p_{2 d-1}\right\}\right)$ such that $h(u) \in\left\{p_{1}, \ldots, p_{2 d-1}\right\}$. Thus, for any choice of $\prod_{j=1}^{d_{h}} \frac{z-b_{j}}{1-\overline{b_{j} z}}$ there are only finitely many possibilities for $\lambda^{\prime}$ which need to be considered, regardless of the choice of $p_{2 d}$. Through all of this, we 
conclude that

$$
\hat{\mathcal{H}}=\bigcup_{p_{2 d} \in A} \mathcal{H}_{f}\left(\left\{\infty, p_{1}, \ldots, p_{2 d}\right\}^{c}\right)
$$

is a finite set of functions, where $A$ is the unit circle minus $\left\{p_{1}, \ldots, p_{2 d-1}\right\}$. Suppose that $\mathcal{H}_{f}\left(\left\{\infty, p_{1}, \ldots, p_{2 d}\right\}^{c}\right) \supsetneq\{f\}$ for all choices of $p_{2 d}$ in $A$. Then there must be a function $k \in \hat{\mathcal{H}}$ not equal to $f$ which lies in $\mathcal{H}_{f}\left(\left\{\infty, p_{1}, \ldots, p_{2 d}\right\}^{c}\right)$ for infinitely many choices of $p_{2 d}$. It must be that $f^{-1}\left(p_{2 d}\right) \cap k^{-1}\left(p_{2 d}\right) \neq \emptyset$ for infinitely many choices of $p_{2 d}$, and it then follows that $k=f$ on infinitely many points. We conclude that $k=f$ identically. This is a contradiction, since it was assumed that $k$ is different from $f$. We conclude that there is some choice of $p_{2 d}$ such that $\mathcal{H}_{f}\left(\left\{\infty, p_{1}, \ldots, p_{2 d}\right\}^{c}\right)=\{f\}$, and we are done.

Corollary. Suppose that $f$ is a rational function of degree $d$ and that there is a circle or line $C$ such that $f^{-1}(C)$ is also a circle or line. Then there is a set $E$ of $2 d+1$ points such that if $U^{c}=E$ then $\mathcal{H}_{f}(U)=\{f\}$.

Proof. Such a function is readily seen to be Möbius equivalent to a Blaschke product.

\section{An explicit example for $z^{n}$}

We now give explicit domains $U_{n}$ where $\mathcal{H}_{z^{n}}\left(U_{n}\right)=\left\{z^{n}\right\}$. Examples have already been given for $n=1,2$, so we assume $n \geq 3$.

Proposition 3. Let $U_{n}^{c}=\left\{\infty, 1, \omega, \omega^{2}, \ldots, \omega^{n-1}, e^{2 \pi i / n^{3}}\right\}$ where $\omega=e^{2 \pi i / n}$, and let $f(z)=z^{n}$. Then $\mathcal{H}_{f}\left(U_{n}\right)=\{f\}$.

Proof. Suppose $h \in \mathcal{H}_{z^{n}}\left(U_{n}\right)$. By the same argument as in Theorem $1, h(\{|z|=$ $1\})=\{|z|=1\}$ and $h^{-1}(\infty)=\{\infty\}$. This means that $h$ is a polynomial, and by the reflection principle we have $h^{-1}(0)=\{0\}$. Thus, $h(z)=c z^{m}$ for some $m$ and some constant $c$ with $|c|=1$. There are exactly $n(n+1)+1=n^{2}+n+1$ points in $f^{-1}\left(U^{c}\right)$, while $h^{-1}\left(U_{n}^{c}\right)$ has at least $m(n+2)-(2 m-2)=m n+2$ points by the Riemann-Hurwitz formula. Since we must have $h^{-1}\left(U_{n}^{c}\right) \subseteq f^{-1}\left(U_{n}^{c}\right)$ we obtain $m n+2 \leq n^{2}+n+1$, which implies $m \leq n$. For two points on the unit circle $a, b$, define $d(a, b)=\frac{1}{2 \pi}|\operatorname{Arg}(a / b)|$, where $\operatorname{Arg}(z)$ is the argument of the point $z$ in the interval $(-\pi, \pi]$. So, for example, $d\left(1, e^{2 \pi i / n^{3}}\right)=1 / n^{3}$. We have

$$
\inf _{u \in h^{-1}(a), v \in h^{-1}(b)} d(u, v)=\frac{1}{m} d(a, b) .
$$

There are therefore points $u$ and $v$ in $h^{-1}\left(U_{n}^{c}\right)$ which are preimages of 1 and $e^{2 \pi i / n^{3}}$ such that $d(u, v)=\frac{1}{n^{3} m}$. Let us refer to the intervals on $\{|z|=1\}$ between neighboring points in $f^{-1}\left(U_{n}^{c}\right)$ as gaps, and let the size of these gaps be the distance between their endpoints, measured in our metric $d$. Applying (4.1) with $f$ in place of $h$ to the configuration of points forming $U_{n}^{c}$ shows that the sizes of the gaps of $f^{-1}\left(U_{n}^{c}\right)$ are $\frac{1}{n^{2}}, \frac{n^{2}-1}{n^{4}}$, and $\frac{1}{n^{4}}$. Furthermore, each gap of size $\frac{1}{n^{4}}$ has a gap of size $\frac{1}{n^{2}}$ on one side of it and a gap of size $\frac{n^{2}-1}{n^{4}}$ on the other. If $m<n$, then we have $\frac{1}{n^{4}}<d(u, v)<\frac{n^{2}-1}{n^{4}}, \frac{1}{n^{2}}$. This contradicts $\{u, v\} \subseteq f^{-1}\left(U_{n}^{c}\right)$, as $d(u, v)$ must be equal to the size of a gap in $f^{-1}\left(U_{n}^{c}\right)$ or to a sum of the sizes of several adjacent gaps. We conclude that $m=n$. 
Thus, $h(z)$ is a rotation of $z^{n}$, and since $U_{n}$ is not invariant under any nontrivial rotations, we conclude that $h=f$.

\section{Applications to the generalized Kobayashi and Carathéodory metrics}

An application of the results given above lies in studying the generalized Kobayashi and Carathéodory metrics, as introduced in [3] and [4]. Indeed, this was the initial motivation for studying this problem. Given two hyperbolic domains $\Omega$ and $X$ with hyperbolic densities $\rho_{\Omega}, \rho_{X}$, let $\mathcal{H}$ denote the family of holomorphic functions from $\Omega$ into $X$. Set

$$
K_{X}^{\Omega}(w)=\inf _{f \in \mathcal{H}, f(z)=w} \frac{\rho_{\Omega}(z)}{\left|f^{\prime}(z)\right|} .
$$

This is known as the generalized Kobayashi density, and is a density on $X$. The classical Kobayashi density is obtained when $\Omega$ is the unit disk $\Delta$.

Set

$$
C_{X}^{\Omega}(z)=\sup _{f \in \mathcal{H}} \rho_{X}(f(z))\left|f^{\prime}(z)\right| .
$$

This is known as the generalized Carathéodory density, and is a density on $\Omega$. Again, the classical Carathéodory density is obtained when $X=\Delta$. The integrated forms of these densities are known as the generalized Kobayashi metric and generalized Carathéodory pseudo-metric, respectively. Many nice and expected properties of $K_{X}^{\Omega}$ and $C_{X}^{\Omega}(z)$ appear in [3] and [4], but the results in Sections 2 through 4 can be used to show that these generalized densities have some properties that are quite different from their classical counterparts. We now demonstrate this through a series of propositions.

Let us begin with $K_{X}^{\Omega}(w)$. Set $f(z)=z^{n}$, and let $X=U_{n}$ be as in Section 4 so that $\mathcal{H}_{f}(X)=\{f\}$. Set $\Omega=f^{-1}(X) . K_{X}^{\Omega}(w)$ is then a density on $X$, and since $\mathcal{H}=\{f\}$ we may explicitly compute

$$
K_{X}^{\Omega}(w)=\inf _{z^{n}=w} \frac{\rho_{\Omega}(z)}{n|w|^{(n-1) / n}} .
$$

In particular, $K_{X}^{\Omega}(0)=\infty$. This "pole" of the density function allows us to show that a number of properties enjoyed by the classical density fail to hold in the generalized case. We will state these results as Propositions 4 through 7 . It is known that the set $\left\{K_{X}^{\Omega}(w)=0\right\}$ is closed for any $\Omega, X$, and a reasonable guess might be that if this set of degeneracy is non-empty then it must be all of $X$. Our example shows, however, that this guess is incorrect.

Proposition 4. It is possible for $K_{X}^{\Omega}$ to be nondegenerate on $X$ but degenerate at certain points of $X$.

Next, it is known that $\rho_{X}(w) \leq K_{X}^{\Omega}(w)$. Our example shows, however, that we cannot obtain an inequality in the opposite direction.

Proposition 5. $\rho_{X}(w)$ and $K_{X}^{\Omega}(w)$ are not equivalent, in general, even when $K_{X}^{\Omega}(w)$ is nondegenerate. That is, there is no constant $M$ such that $K_{X}^{\Omega}(w) \leq$ $M \rho_{X}(w)$. 
Since $\int_{0}^{\varepsilon} r^{-(n-1) / n} d r<\infty$, the distance between 0 and any point in the generalized Kobayashi metric is finite in this case. Nevertheless, if we do the standard thing in defining the area of any open set $B$ as

$$
\operatorname{Area}(B)=\int_{B}\left(K_{X}^{\Omega}(w)\right)^{2} d A(w)
$$

then upon converting to polar coordinates in a neighborhood of 0 , we see that Area $(B)$ is infinite if $n \geq 3$ and $0 \in B$. We obtain

Proposition 6. It is possible for a compact set in $X$ to have infinite area when measured in terms of the density $K_{X}^{\Omega}(w)$.

To motivate our next example let us consider the hyperbolic metric in the punctured unit disk $\Delta^{*}=\Delta \backslash\{0\}$. We can find a sequence of sets in $\Delta^{*}$ of the form $B(b, r)=\left\{a: \rho_{\Delta^{*}}(b, a)<r\right\}$, which we refer to as balls, whose radii approach 0 and each of which are not homeomorphic to a disc. This can be verified by showing that the hyperbolic length of the semicircle between $\varepsilon$ and $-\varepsilon$ goes to 0 as $\varepsilon \rightarrow 0$, using the formula $\rho_{\Delta^{*}}(z)=\frac{1}{2|z| \log (1 /|z|)}($ see $[4])$.

Let us contrast this with the generalized Kobayashi metric $K_{X}^{\Omega}(a, b)$, which is defined to be

$$
K_{X}^{\Omega}(a, b)=\inf \int_{\gamma} K_{X}^{\Omega}(\gamma(t))|d \gamma|
$$

where the infimum is taken over all curves connecting $a$ and $b$. For $\varepsilon$ very small we have

$$
K_{X}^{\Omega}(0, \varepsilon) \approx \rho_{\Omega}(0) \int_{0}^{\varepsilon} \frac{1}{n r^{(n-1) / n}} d r=\rho_{\Omega}(0) \varepsilon^{1 / n}
$$

whereas, by considering the curve $\gamma(t)=\varepsilon e^{i t}, t \in[0, \pi]$, we have

$$
K_{X}^{\Omega}(-\varepsilon, \varepsilon) \leq(1+\delta) \rho_{\Omega}(0) \int_{0}^{\pi} \frac{1}{n \varepsilon^{(n-1) / n}} \varepsilon d t=(1+\delta) \frac{\pi}{n} \rho_{\Omega}(0) \varepsilon^{1 / n}
$$

for some $\delta>0$ which can be made arbitrarily small as $\varepsilon \rightarrow 0$. We see that for $n \geq 4$ and $\varepsilon$ sufficiently small, we have $K_{X}^{\Omega}(0, \varepsilon)>K_{X}^{\Omega}(-\varepsilon, \varepsilon)$. This shows that we may find a sequence of balls with radii measured in $K_{X}^{\Omega}$ which are centered at the points $\frac{1}{n}$, whose radii approach 0 , and each of which are not homeomorphic to a disc. This is similar to what we observed in the hyperbolic case, but there is a difference. The sequence of hyperbolic balls in $\Delta^{*}$ approaches the boundary point 0 , and this turns out to be indicative of the general behavior of the hyperbolic density in this context. It is not hard to show that for any compact set $K$ in a hyperbolic domain $V$ there is a $\beta>0$ such that any hyperbolic ball centered in $K$ with radius less than $\beta$ is homeomorphic to a disc. To put it informally, sufficiently small hyperbolic balls away from the boundary of a domain are simply connected. Our example shows that this fails to hold for $K_{X}^{\Omega}$, since the given balls approach 0 , which is an interior point of $X$. We therefore obtain

Proposition 7. It is possible for a sequence of balls in the metric $K_{X}^{\Omega}$ which are not homeomorphic images of discs to stay within a compact set in $X$ and have radii which approach 0. 
Now let us consider $C_{X}^{\Omega}$ with the same choices of $\Omega$ and $X$. As $\mathcal{H}=\left\{z^{n}\right\}$ we find

$$
C_{X}^{\Omega}(z)=\rho_{X}\left(z^{n}\right) n|z|^{n-1} .
$$

Again it is known that the set $\left\{C_{X}^{\Omega}(z)=0\right\}$ is closed, and this set of degeneracy may be all of $\Omega$. Our example shows, however, that

Proposition 8. It is possible for $C_{X}^{\Omega}$ to be nondegenerate on $\Omega$ but degenerate at certain points of $\Omega$.

It is known that $\rho_{\Omega}(w) \geq C_{X}^{\Omega}(w)$. Again we see from our example that

Proposition 9. $\rho_{\Omega}(w)$ and $C_{X}^{\Omega}(w)$ are not equivalent, in general, even when $C_{X}^{\Omega}(w)$ is nondegenerate. That is, there is no constant $M$ such that $\rho_{\Omega}(w) \leq$ $M C_{X}^{\Omega}(w)$.

Finally, we have

$$
\frac{\rho_{\Omega}\left(w, w^{\prime}\right)}{\left|w-w^{\prime}\right|} \rightarrow \rho_{\Omega}(w) \text { as } w^{\prime} \rightarrow w
$$

If $C_{X}^{\Omega}(a, b)$ is the generalized Kobayashi metric on $\Omega$, defined analogously to (5.5), then we have for $w^{\prime}$ close to 0

$$
C_{X}^{\Omega}\left(0, w^{\prime}\right) \approx \rho_{X}(0) \int_{0}^{\left|w^{\prime}\right|} n r^{n-1} d r=\rho_{X}(0)\left|w^{\prime}\right|^{n} .
$$

This leads to

\section{Proposition 10.}

$$
\frac{C_{X}^{\Omega}\left(0, w^{\prime}\right)}{\left|w^{\prime}\right|^{m}} \rightarrow 0 \quad \text { as } \quad w^{\prime} \rightarrow 0
$$

for $m<n$, and

$$
\frac{C_{X}^{\Omega}\left(0, w^{\prime}\right)}{\left|w^{\prime}\right|^{n}} \rightarrow \rho_{X}(0) \text { as } w^{\prime} \rightarrow 0 .
$$

\section{Further questions}

The technique given in Section 3 seems not to extend directly to arbitrary rational functions. This is because the key lay in showing that any map in $\mathcal{H}_{f}(U)$ must be of a specific kind, and this seems more difficult to do with arbitrary maps. Nevertheless, we venture the following conjecture.

Conjecture. If $f$ is a rational function of degree $d$, there is a domain $U$ where $U^{c}$ has $2 d+1$ points and $\mathcal{H}_{f}(U)=\{f\}$.

The reason for guessing that $2 d+1$ points suffice is that this is enough points to rule out all maps of degree greater than $d$, as is shown in the beginning of the proof of Theorem 1. The examples given in Section 4 make do with only $d+2$ points, whereas the remarks in Section 2 show that at least 5 points are required in all cases. It may be interesting to find the optimal domains which work for a given $f$, where optimality is measured in terms of the size of the boundaries.

Another potentially interesting problem is to find a domain $U$ with $\mathcal{H}_{f}(U)=\{f\}$, where $f$ is now a non-rational entire function. Picard's Big Theorem (see [1]) shows that if $U^{c}$ has three or more points then $V^{c}$ must be infinite, complicating matters. The results for rational functions were deduced by first eliminating all but a relatively 
small number of potential maps between domains. Perhaps something similar could be done with entire functions satisfying certain conditions, for instance for functions of finite order (see [2]).

Acknowledgements. I'd like to thank Linda Keen, Ross Flek, Fred Gardiner, Nikola Lakic and Nils Tongring for helpful conversations, as well as an anonymous referee for a careful reading. I am also grateful for support from Australian Research Council Grant DP0988483.

\section{References}

[1] Conway, J. B.: Functions of one complex variable. - Springer-Verlag, 1978.

[2] BoAs, R. P.: Entire functions. - Academic Press, 1954.

[3] Keen, L., and N. LaKic: A generalized hyperbolic metric for plane domains. - In: In the tradition of Ahlfors-Bers IV, Proceedings of the Ahlfors-Bers Colloquium (May 19-22, 2005, University of Michigan, Ann Arbor, Michigan), Contemp. Math. 432, Amer. Math. Soc., Providence, RI, 2007, 107-118.

[4] Keen, L., and N. LAKIC: Hyperbolic geometry from a local viewpoint. - Cambridge Univ. Press, 2007.

Received 6 July 2011 